\title{
Influence de l'anthropisation sur la structure de quatre espèces utilitaires dans le Chantier d'Aménagement Forestier de Cassou, Burkina Faso
}

\author{
François Wenemi KAGAMBEGA ${ }^{1, *}$, Abel KADEBA ${ }^{2}$, Nouhoun ZAMPALIGRE ${ }^{3}$, \\ Bernadette ZONGO / NITIEMA ${ }^{4}$, Louis SAWADOGO ${ }^{1}$ et Joseph Issaka BOUSSIM ${ }^{4}$ \\ ${ }^{I}$ Centre National de la Recherche Scientifique et Technologique, Institut de l'environnement et de Recherches \\ Agricoles, Département Environnement et Forêts, 03 BP 7047, Ouagadougou 03, Burkina Faso. \\ ${ }^{2}$ Univerisité de Dédougou, Institut des Sciences de l'environnement et du Développement Rural, BP 176 \\ Dédougou, Burkina Faso. \\ ${ }^{3}$ Centre National de la Recherche Scientifique et Technologique, Institut de l'environnement et de Recherches \\ Agricoles, Département Productions animales, 03 BP 7047, Ouagadougou 03, Burkina Faso. \\ ${ }^{4}$ Université Joseph Ki-Zerbo, Laboratoire de Biologie et Ecologie Végétales, 03BP 7021 Ouagadougou 03, \\ Burkina Faso. \\ *Auteur correspondant ; E-mail: wenemifrancois@yahoo.fr; Tél: (+226) 70747578.
}

Les auteurs remercient le Center for International Forestry Research (CIFOR) qui a financé cette étude.

\section{RESUME}

La compréhension de la dynamique des espèces dans les Chantiers d'Aménagement Forestiers (CAF) est primordiale pour leur conservation durable. L'objectif de cette étude est d'évaluer l'effet de l'anthropisation sur la dynamique de quatre espèces utilitaires (Vitellaria paradoxa, Burkea africana, Crossopteryx febrifuga et Detarium microcarpum) dans le CAF de Cassou. Pour ce faire, un inventaire dendrométrique a été réalisé par la méthode d'échantillonnage de Whittaker dans les Unités d'Aménagement Forestiers (UAFs) 1, 6, 8 et 9 où les incidences des activités agricoles, de la coupe de bois, du pâturage et des feux tardifs ont été évaluées. Les résultats montrent qu'en dehors des activités agricoles qui ont été moins fréquentes, les UAFs ont été très touchées par le feu (> 55\%), la coupe de bois $(>60 \%)$ et le pâturage (> 85\%). L'analyse structurale des espèces indique des densités plus élevées dans les UAFs 1 et 8 . Seul Detarium microcarpum présente une structure stable dans toutes les UAFs tout comme Vitellaria paradoxa dans les UAFs 1, 6 et 8 . A part Detarium microcarpum, les trois autres espèces ont des difficultés de régénération. La mise en œuvre d'un programme de conservation de ces espèces dans le CAF est donc nécessaire pour protéger et faciliter la croissance de leurs juvéniles.

(C) 2019 International Formulae Group. All rights reserved.

Mots clés: Facteurs anthropiques, aménagement forestier, diversité structurale, Burkina Faso.

\section{Effect of anthropogenic factors on the structure and dynamics of four useful species in the forest management domains (FMD) of Cassou (Burkina Faso)}

\author{
ABSTRACT \\ Understanding the structural dynamics of woody species in the Forest Management Centre (FMC) is \\ necessary for their sustainable conservation. The aim of this study is to assess the effect of anthropogenic
}


factors on the dynamics of four useful species (Vitellaria paradoxa, Burkea africana, Crossopteryx febrifuga, Detarium microcarpum) in the FMC of Cassou. To this end, dendrometric measures using the Whittaker sampling method were carried out in the Forest Management Units (FMUs) 1, 6, 8 and 9, in which anthropogenic factors such as practice of agriculture, logging, grazing and fire were evaluated. The results show that apart from agricultural activities, which were relatively less frequent, the FMUs were very affected by fire $(>55 \%)$, tree cutting $(>60 \%)$ and livestock grazing $(>85 \%)$. Structural analysis of species showed higher densities in FMUs 1 and 8. Detarium microcarpum showed a stable structure in all the FMUs and Vitellaria paradoxa was only stable in the FMUs 1, 6 and 8. Except for Detarium microcarpum, the overall regeneration' rate of the assessed species was weak. Therefore, implementation of conservation program in the FMC of Cassou is needed to protect and facilitate the growth of the juveniles of these species.

(C) 2019 International Formulae Group. All rights reserved

Keywords: Anthropogenic factors, forest management, structural diversity, Burkina Faso.

\section{INTRODUCTION}

La zone soudanienne ouest-africaine connaît depuis plusieurs décennies, une crise écologique récurrente qui se manifeste par une profonde modification des différents ensembles écologiques en général et les formations végétales en particulier. Les causes de la transformation de ces formations végétales sont diverses et traduisent des situations parfois spécifiques à un pays, ou à des groupes de pays (Sorg, 2000). Au Burkina Faso, comme dans les autres pays du Sahel, les ressources forestières jouent un rôle fondamental dans la satisfaction des besoins de base des communautés locales et dans le développement socioéconomique du pays. Cependant ces ressources subissent de nos jours une exploitation intense compromettant leur capacité à couvrir les besoins des populations (FAO, 2015). Les facteurs majeurs de dégradation des ressources forestières sont les feux de brousse, la coupe abusive du bois, le surpâturage et la mortalité naturelle des espèces due aux aléas climatiques (Sakpota et Odén Per, 2009; Gbedahi et al., 2019).

La nécessité de sauvegarder les ressources ligneuses, tout en satisfaisant les besoins quotidiens des populations, est une préoccupation majeure aussi bien pour l'Etat, les ONG, les chercheurs que pour les autres acteurs socio-culturels et économiques locaux. L'aménagement des forêts naturelles est l'une des solutions à envisager. Ainsi, depuis les années 1980, l'état burkinabé s'est doté progressivement d'un cadre juridique et institutionnel concernant l'aménagement des formations naturelles pour aboutir à la mise en œuvre des Chantiers d'Aménagement Forestiers (CAF) sur financement du PNUD avec une implication de la FAO (CoulibalyLingani et al., 2011). L'objectif principal de ces CAF avec des Unités d'Aménagement Forestiers (UAFs) installées au Sud du pays, était de réguler l'exploitation du bois-énergie au profit des villages environnants et des centres urbains du pays. L'aménagement des forêts naturelles devrait aussi bien contribuer à générer de nouvelles sources de revenus pour les populations et assurer la conservation de la biodiversité (MEDD, 2011). Malheureusement, des pressions croissantes sont exercées sur ces écosystèmes forestiers à travers des collectes intensives de produits forestiers ligneux et non-ligneux, du fait de la croissance démographique et de l'accroissement de la valeur marchande des produits dérivés des essences forestières. Bien que le modèle des CAF au Burkina Faso fonctionne sur la base d'un plan d'aménagement et de gestion approuvée, les pratiques d'aménagement se heurtent à l'insuffisance de connaissances sur la biologie et l'écologie des espèces locales, ainsi que des paramètres de productivité des formations naturelles sous l'influence des différents facteurs biotiques et abiotiques (Sawadogo, 2009). Des études antérieures ont porté sur les défis de la gestion décentralisée des forêts, les facteurs influant sur l'accès aux produits forestiers, la gestion participative ou conjointe des forêts aménagées (Bouda et al., 2011; Coulibaly-Lingani et al., 2014). Aussi, une étude récente réalisée au Burkina Faso suscite 
des inquiétudes quant à la durabilité du CAF au regard des besoins grandissants des populations humaines (Arevalo, 2016) et de la forte pression anthropique exercée sur un certain nombre d'espèces surexploitées pour le bois de chauffe et de service (Tanyi et al., 2018). Parmi les espèces ligneuses d'importance majeure exploitées dans le CAF, Vitelleria paradoxa C. F. Gaertn., Detarium microcarpum Guill. et Perr., Burkea africana Hook. et Crossopteryx febrifuga Benth., subissent une forte pression anthropique (Sawadogo, 2009). En plus de leur exploitation pour le bois énergie, ces espèces occupent une place socio-économique importante dans la vie quotidienne des populations (Sereme et al., 2008 ; Kouyaté et Lamien, 2011; Dro et al., 2013) car elles sont privilégiées dans plusieurs catégories d'usage (alimentation, cosmétique, pharmacopée, artisanat, etc.). Cependant, l'importance vitale des produits fournis par les espèces utilitaires, est aussi une cause majeure de leur destruction à cause de l'exploitation intense (Ouédraogo et al., 2006). Étant donné que toutes les unités d'aménagements forestiers (UAFs) du CAF de Cassou (en exploitation depuis 1991) se trouvent actuellement à des stades différents de leur deuxième cycle de rotation qui sera atteint en 2021, il devient impératif d'analyser l'impact des perturbations anthropiques en se basant sur la structure des espèces exploitées. La connaissance de la structure des populations de ces espèces de grande importance apparaît nécessaire pour mieux appréhender leur statut ainsi que le niveau de menace pour proposer des stratégies de gestion durable.

L'objectif général de la présente étude est d'évaluer l'impact des perturbations anthropiques sur la structure de quatre (4) espèces ligneuses utilitaires du CAF de Cassou que sont Vitelleria paradoxa, Detarium microcarpum, Burkea africana et Crossopteryx febrifuga. De façon spécifique, il s'agit: (i) de caractériser l'état des perturbations anthropiques (pâturage, feu, pratiques agricoles et coupe de bois) dans les unités d'aménagements forestiers sélectionnées et (ii) de déterminer la structure des peuplements de ces espèces et caractériser leur tendance évolutive. L'étude se fonde sur les hypothèses suivantes : (H1) La végétation des UAFs proches de l'unité de gestion est moins exploitée et donc plus perturbée que celle des UAFs éloignées; (H2) Les populations des quatre espèces ciblées présentent une dynamique structurale stable dans les UAFs proches de l'unité de gestion. Les résultats obtenus serviront à l'élaboration de stratégies de gestion durable de la biodiversité afin d'orienter les décideurs publics et les acteurs de développement, dans la planification et la prise de décisions.

\section{MATERIEL ET METHODES}

\section{Présentation de la zone d'étude}

L'étude a été menée dans le Chantier d'Aménagement Forestier (CAF) de Cassou, situé à $35 \mathrm{~km}$ de Sapouy, chef-lieu de la province du Ziro (Figure 1). Localisé dans le domaine phytogéographique Sud-soudanien, entre les latitudes $12^{\circ} 24^{\prime}$ et $12^{\circ}$ Nord et les longitudes $2^{\circ} 39^{\prime}$ et $2^{\circ}$ Ouest, le CAF de Cassou est sous l'influence d'un climat soudanien caractérisé par une longue saison sèche (octobre à avril) et une saison pluvieuse qui dure généralement moins de 5 mois (de mai à septembre / octobre). Les précipitations annuelles peuvent atteindre ou même dépasser $800 \mathrm{~mm}$ suivant les années tandis que les températures moyennes mensuelles se situent entre $35{ }^{\circ} \mathrm{C}$ en avril et $25{ }^{\circ} \mathrm{C}$ en décembre (Etongo et al., 2018). La zone appartient au vaste complexe du plateau mossi mais présente un relief peu accidenté se caractérisant par une vaste pénéplaine. Les sols sont de types argileux- sableux, limoneux ou gravillonnaires et sont représentés par les Cambisols, les Lixisols et les Luvisols (Driessen et al., 2001).

\section{Historique et prescriptions d'aménagement du CAF de Cassou}

D'une superficie de 29.515 ha, le CAF de Cassou fait partie d'un ensemble de sept (7) CAFs du Burkina Faso créés en 1986 dans un programme d'aménagement des formations naturelles sur financement PNUD avec une implication de la FAO (Etongo et al., 2018). 
Dans le but de parvenir à un aménagement durable des formations végétales sur le terrain, les CAF du Burkina Faso ont été divisées en unités et sous-unités par les acteurs en charges de sa gestion. Ainsi, le CAF de Cassou qui a été créé en 1991, compte neuf (9) Unités d'Aménagement Forestiers (UAFs) et trois (03) sous-unités. Chaque sous-unité ou unité du CAF de Cassou est subdivisée en quinze (15) parcelles qui seront exploitées successivement chaque année avec un cycle de rotation de 15 ans. L'exploitation suit le schéma des prescriptions suivantes :

- une coupe sélective de $50 \%$ du volume de bois commercialisable avec 15 ans de rotation;

- l'utilisation du feu précoce annuel sauf sur les parcelles nouvellement coupées ;

- la protection des parcelles exploitées du feu et du pâturage pendant 3 à 5 ans ;

- l'interdiction du pâturage dans les forêts classées non aménagées, les parcs nationaux et les ranchs de gibier;

- l'enrichissement par semis direct et par plantation des espaces exploitées et des zones dénudées avec des espèces locales.

Le CAF de Cassou a été choisie en raison de la présence d'une unité de gestion toujours en activité, ce qui constitue une meilleure opportunité pour tester les hypothèses énumérées en liens avec l'efficacité des approches de gestion.

\section{Collecte de données}

\section{Echantillonnage et installation des placettes}

Après une prospection des paysages du Chantier d'Aménagement Forestier (CAF) de Cassou, quatre (04) Unités d'Aménagement Forestiers (UAFs) sur un total de douze (12) UAF ont été choisis selon des critères tels que la présence des quatre espèces sélectionnées, la distance avec l'unité de gestion du CAF, la représentativité, l'homogénéité et la géomorphologie. Ainsi, les UAFs étudiées que sont l'UAF 1, l'UAF 6, l'UAF 8 et l'UAF 9, ont été sélectionnées à l'aide de cartes d'occupation $\mathrm{du}$ sol préexistantes. Cet échantillonnage visait à couvrir toutes les unités géomorphologiques et les types physionomiques du peuplement existant dans le CAF.

L'étude a été conduite sur l'ensemble des 15 parcelles de chacune des UAFs ciblées où un échantillonnage stratifié et aléatoire a été adopté; l'unité d'échantillonnage qui représente un relevé floristique est une placette de $1000 \mathrm{~m}^{2}$. L'installation des placettes a tenu compte de la taille des UAFs avec un taux de sondage de $0,3 \%$ comme cela est présenté dans le Tableau 1. Sur cette base, les points représentant les placettes de chaque parcelle dans les différentes UAFs ont été prépositionnés de façons aléatoires sur les cartes géo-référencier des UAFs et les coordonnées géographiques reportées dans des GPS. Les déplacements pour l'implantation des placettes d'inventaire sur le terrain se sont effectués à l'aide de ces GPS, à travers les pistes existantes.

\section{Caractérisation de la pression anthropique}

Les facteurs de perturbations dus à l'action de l'homme que sont les feux tardifs, les activités agricoles, le pâturage et la coupe frauduleuse de bois, ont été caractérisés dans chaque placette. La pression anthropique est nulle dans une placette, lorsqu'il n'y a ni trace de feu, ni trace de pâture d'animaux domestiques (présence d'animaux en pâture, de déjections, de traces de piétinements, etc.) ni activité agricole, ni trace de coupe récente de bois. Par contre, lorsque dans une placette, on observe l'un de ces phénomènes, c'est la superficie touchée, qui détermine le degré de la pression anthropique (Agbani et al., 2018). Ainsi la pression anthropique a été évaluée à l'échelle de chaque placette selon une codification à quatre niveaux pour chaque facteur :

- lorsqu'aucune trace de perturbations anthropiques relatives aux facteurs ci-dessus énumérés n'est observée dans une placette, le code 0 est attribué ;

- lorsque les traces sont faibles $(<10 \%$ de la placette), le code 1 est attribué,

- le code 2 est attribué lorsque le degré de perturbation est moyen (entre $10 \%$ et $40 \%$ );

- lorsque les traces de perturbation sont élevées (> $40 \%$ de la placette), le code 3 est attribué. 


\section{Inventaire floristique et mesures dendrométriques}

La méthode retenue pour la collecte des données floristiques et dendrométriques a été celle de l'échantillonnage à surface variable développée par Whittaker (1965) modifiée par Stohlgren et al. (1995). Il s'agit d'une méthode d'inventaire basée sur une parcelle principale (placette $\mathrm{D}$ ) rectangulaire de $1000 \mathrm{~m}^{2}(50 \mathrm{~m} \times 20 \mathrm{~m})$ contenant une sousparcelle $C$ de $100 \mathrm{~m}^{2}(20 \mathrm{~m} \times 5 \mathrm{~m})$ et deux sous-parcelles B de $10 \mathrm{~m}^{2}$ chacune (Figure 2).

L'inventaire a été effectué consécutivement dans les deux sous-placettes $\mathrm{B}$, la sous-placette C et la placette D. Dans les sous-placettes $\mathrm{B}$, seuls les individus de DBH (diamètre du tronc à hauteur de poitrine ou à $1,30 \mathrm{~m}$ du sol) $<5 \mathrm{~cm}$, c'est-à-dire comptés dans la régénération potentielle (Ouedraogo et al., 2006), ont été identifiés et inventoriés dans le but d'apprécier l'état de renouvellement naturel des espèces ligneuses et leur dynamisme après exploitation. Les individus à bois non exploitables $(5 \mathrm{~cm} \leq$ DBH $<10 \mathrm{~cm})$ ont été inventoriés dans la sous-placette $\mathrm{C}$ tandis que les individus adultes à bois exploitables $(\mathrm{DBH} \geq 10 \mathrm{~cm})$ ont été inventoriés dans la placette D. L'inventaire floristique des espèces ligneuses a été faite de façon exhaustive dans les différentes placettes et sous-placettes. L'identification des échantillons d'espèces récoltées sur le terrain a été faite par comparaison avec les spécimens de l'Herbier National du Burkina Faso. La nomenclature botanique utilisée a été celle du groupe de la classification phylogénétique des angiospermes (APG III, 2009). Pour chaque individu inventorié, les paramètres suivants ont été mesurés : le DBH et la hauteur. Une perche graduée de $3 \mathrm{~m}$ et un dendromètre électronique associé au télémètre laser ont servi à l'estimation de la hauteur des arbres, depuis la base du pied jusqu'au bout de la branche la plus élevée.

\section{Analyse et traitement des données Pression anthropique}

La pression anthropique a été évaluée pour chaque facteur en considérant la proportion des placettes concernée par le facteur de perturbation codifié par rapport au nombre total de placettes inventoriées dans l'UAF selon la formule suivante :

Placettes affectées par le code i

$$
\frac{\text { Nombre de placettes codifiées i }}{\text { Nombre total de placettes inventoriées }}
$$

Où i représente le niveau du code $(0,1,2$ ou 3) pour le facteur concerné.

\section{Données floristiques et dendrométriques}

Afin d'apprécier la dynamique et les tendances évolutives des quatre espèces (Burkea africana, Crossopteryx febrifuga, Detarium microcarpum et Vitellaria paradoxa) ciblées dans les UAFs étudiées, la densité, la surface terrière, les moyennes des hauteurs et des diamètres à hauteur de poitrine $(\mathrm{DBH})$ de ces espèces ainsi que le taux de régénération, ont été calculés à partir des données de terrain.

Le taux de régénération $(\mathrm{Tr})$ de chaque espèce a été calculé par la formule suivante :

$$
\begin{array}{r}
\operatorname{Tr}=\frac{\text { Nombre d'individus de diamètre }<5 \mathrm{~cm}}{\text { Nombre total d'individus présents dans l'UAF }} \\
\times 100
\end{array}
$$

À partir d'un seuil de $10 \mathrm{~cm}$, des classes de diamètre d'amplitude $5 \mathrm{~cm}$ ont été constituées afin d'établir la structure de chaque espèce étudiée (Burkea africana, Crossopteryx febrifuga, Detarium microcarpum et Vitellaria paradoxa) à l'aide d'histogrammes de distribution diamétrales. Une distribution théorique suivant la loi de Weibull a été réalisée à l'aide du logiciel MINITAB 14, pour caractériser la structure des populations de chaque espèce. $\mathrm{La}$ distribution de Weibull à trois paramètres (de position a, d'échelle ou taille $b$ et de forme $c$ ) a été utilisée selon la formule:

$$
f(x)=\frac{c}{b}\left(\frac{x-a}{b}\right)^{c-1} \exp \left[-\left(\frac{x-a}{b}\right)^{c}\right]
$$

$x=$ diamètre des arbres et $f(x)=$ valeur de densité de probabilité au point $x$.

Le paramètre « $\mathrm{a}$ » correspond à la valeur seuil, c'est-à-dire à la plus petite valeur de diamètre retenue pour la constitution des histogrammes. Le paramètre «b » est lié à la 
valeur centrale de la distribution des classes de diamètre. Le paramètre $\langle\mathrm{c} »$ traduit la structure observée et selon sa valeur (Tableau 2), détermine la forme de la distribution structurale des peuplements.

Le choix de la distribution de Weibull a été fait à la faveur de sa souplesse d'emploi et de la grande variabilité de formes de distribution qu'elle produit (Aboubacar et al., 2018). Elle permettrait dans le contexte de l'étude d'apprécier l'état des peuplements de chacune des espèces ciblées et d'identifier celles qui sont les plus affectées par les facteurs anthropiques.

\section{Analyses statistiques}

Les tests de Shapiro-Wilk et de Levene ont été réalisés pour tester respectivement la normalité et l'égalité des variances de chaque paramètre dendrométrique des espèces étudiées (hauteur, densité, diamètre et taux de régénération) au sein des UAFs. Lorsque les hypothèses de normalité et d'égalité de variance étaient vérifiées, une analyse de variance à un facteur a été effectuée pour évaluer la différence de chaque paramètre entre les UAFs. Lorsque les moyennes étaient significativement différentes et les effectifs suffisants, un test de comparaison multiple était appliqué au seuil de 5\%; le test de Tukey (HSD: Honestly Significant Difference). Le logiciel R (R Development Core Team, 2008) a permis d'effectuer ces tests statistiques.

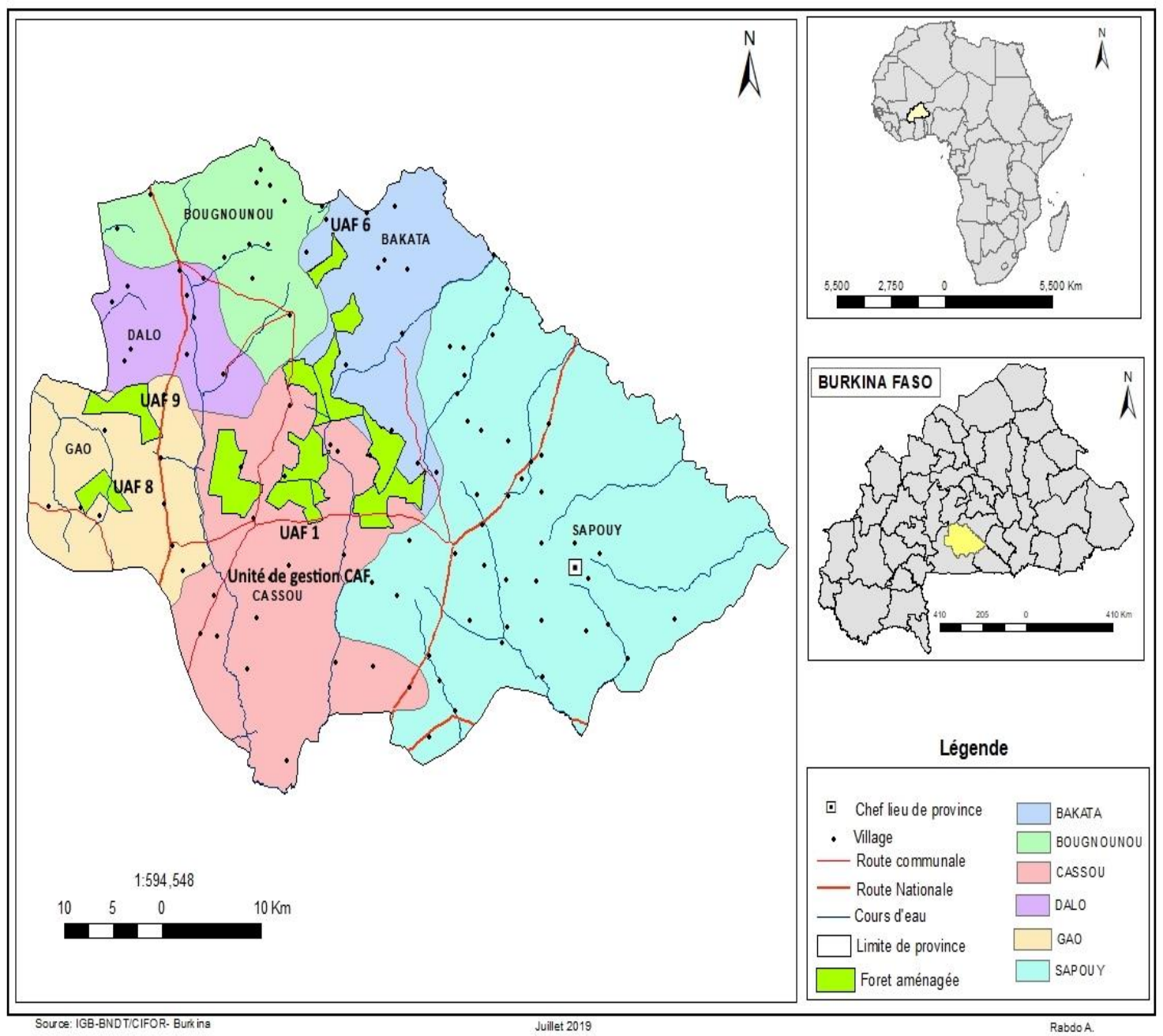

Figure 1 : Carte de localisation du CAF de Cassou et des UAFs étudiées. 
Tableau 1: Caractéristiques des UAFs étudiées et répartition du nombre de placettes.

\begin{tabular}{ccccc}
\hline Caractéristiques des UAFs & UAF 1 & UAF 8 & UAF 9 & UAF 6 \\
\hline Quelques villages associés & Cassou & $\begin{array}{c}\text { Mao-Nassira, } \\
\text { Gao, Tekourou }\end{array}$ & $\begin{array}{c}\text { Dalo, Gao, } \\
\text { Dao, Passin }\end{array}$ & $\begin{array}{c}\text { Zinoua, } \\
\text { Lérou }\end{array}$ \\
Distance avec l'unité de gestion (Km) & 3 & 8 & 10 & 15 \\
Superficie (ha) & 2093 & 1865 & 3277 & 1148 \\
Superficie moyenne de la parcelle (ha) & 139,53 & 124,33 & 218,46 & 76,53 \\
Nombre de placettes / parcelle & 5 & 4 & 7 & 3 \\
Nombre total de placettes & 75 & 60 & 105 & 45 \\
\hline
\end{tabular}

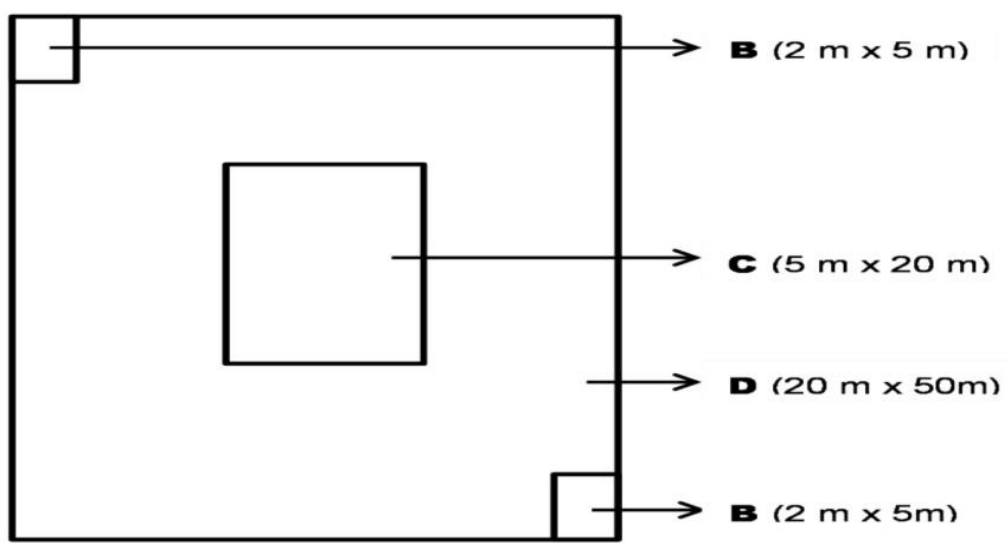

Figure 2: Schéma réadapté d'une placette de Whittaker.

Tableau 2: Interprétation des distributions selon le modèle de Weibull (Glèlè Kakaï et al., 2016).

\begin{tabular}{ll}
\hline $\begin{array}{l}\text { Valeurs du } \\
\text { paramètre c }\end{array}$ & Interprétation de la distribution \\
\hline $\mathrm{c}<1$ & $\begin{array}{l}\text { Distribution en J-renversé, caractéristique des peuplements multispécifiques à fort } \\
\text { potentiel de régénération. }\end{array}$ \\
& $\begin{array}{l}\text { Distribution exponentiellement décroissante, caractéristique de la population à } \\
\text { relativement fort potentiel de régénération mais présentant un problème de survie } \\
\text { lors de la transition entre les stades de développement. }\end{array}$ \\
& $\begin{array}{l}\text { Distribution asymétrique positive ou asymétrique droite, caractéristique des } \\
\text { peuplements artificiels monospécifiques avec prédominance relative d'individus }\end{array}$ \\
$1<\mathrm{c}<3,6$ & $\begin{array}{l}\text { jeunes et de faible diamètre. Elle peut aussi être caractéristique de populations à } \\
\text { faible potentiel de régénération due aux actions exogènes surtout dans les petites } \\
\text { classes de diamètre. }\end{array}$ \\
& $\begin{array}{l}\text { Distribution symétrique, structure normale, caractéristique des populations } \\
\text { d'espèce à faible potentiel de régénération due aux actions exogènes ou } \\
\mathrm{c}=3,6\end{array}$ \\
& $\begin{array}{l}\text { caractéristiques de l'espèce. } \\
\text { Distribution asymétrique négative ou asymétrique gauche, caractéristique des }\end{array}$ \\
$\mathrm{c}>3,6$ & $\begin{array}{l}\text { Elle peut aussi être caractéristique des populations dégradées à très faible } \\
\text { potentiel de régénération dû aux pressions anthropiques et peut indiquer des } \\
\text { populations en extinction. }\end{array}$ \\
\hline
\end{tabular}




\section{RESULTATS}

\section{Incidences des perturbations anthropiques dans les UAFs étudiées}

La Figure 3 présente l'ampleur des différents facteurs anthropiques dans les différentes UAFs. L'analyse de ces résultats montre que toutes les unités parcourues présentent des traces de ces facteurs dans des proportions variables suivant les UAFs en lien avec leur emplacement. L'UAF 1 est très faiblement touchée par les activités agricoles (1\%) pendant que les trois autres UAFs présentent des traces de cultures avec des incidences moyennes à élevés même si l'UAF 8 est moins perturbée (7\%) par rapport aux UAFs 6 et 9 qui sont touchées dans des proportions de $29 \%$ et $34 \%$ respectivement.

Les coupes récentes de bois ont été observées dans toutes les UAFs étudiées dans des proportions allant de $60 \%$ à $73 \%$ des placettes inventoriées. Les UAFs 9 et 1 sont les plus touchées (>70\%) avec une incidence plus élevée dans l'UAF 9 tandis que l'UAF 6 présente la plus faible proportion $(60 \%)$.

Les indices de pâturage ont été également caractérisés dans toutes les UAFs étudiées dans des proportions très élevées (> $85 \%$ ) avec toutefois des intensités variables suivant l'emplacement des UAFs. L'UAF 1 est le plus perturbée avec toutes ces placettes présentant des indices de pâturage, suivi par l'UAF 8 (95\%), l'UAF 9 (89\%) et l'UAF 6 (87\%). En termes d'incidence, les UAFs 1 et 8 présentent les intensités les plus élevées (> $60 \%)$.

Toutes les unités parcourues présentent des traces de feu dans des proportions allant de 55\% à 77\% des placettes inventoriées. Les UAFs 1 et 6 sont les plus touchés suivi respectivement par les UAF 8 et 9 ; toutefois, l'ampleur du feu est plus importante dans les UAF 8 et 1 avec plus de $30 \%$ de leurs placettes présentant une incidence élevée ; viennent ensuite l'UAF 6 et l'UAF 9 avec respectivement $28,8 \%$ et $23,8 \%$ de placettes touchées pour la même incidence.

\section{Structures démographiques et capacité de régénération des espèces}

Les caractéristiques structurales des peuplements des quatre espèces montrent des valeurs très variables suivant les différentes UAFs (Tableau 3). L'analyse statistique montre que la densité de chaque espèce varie significativement suivant les UAFs $(\mathrm{p}<0,05)$ avec des valeurs généralement plus élevées dans les UAFs 1 et 8 , contrairement aux autres variables (Diamètre moyen et Hauteur moyenne). Pour chacune des quatre espèces, la densité des peuplements révèle l'importance majeure d'individus de diamètre faible $(\mathrm{DBH}<5 \mathrm{~cm})$ suivi généralement par les individus de diamètre moyen $(5 \mathrm{~cm} \leq$ $\mathrm{DBH}<10 \mathrm{~cm}$ ) puis ceux à gros diamètre $(\mathrm{DBH} \geq 10 \mathrm{~cm})$, dans les UAFs 1 et 8 . Les UAFs 6 et 9 présentent une situation différente avec une prédominance des individus à gros diamètre de Vitellaria paradoxa et de Burkea africana dans l'UAF 6 et une prédominance des individus de diamètre moyen pour Detarium microcarpum et Vitellaria paradoxa respectivement dans l'UAF 6 et l'UAF 9. Cependant, dans ces deux dernières UAFs, Crossopteryx febrifuga présente la même tendance que dans les UAFs 1 et 8 .

Les résultats relatifs au potentiel de régénération des espèces sont reportés dans le Tableau 4. A l'exception de l'UAF 6, les individus jeunes de l'ensemble des espèces sont bien représentés dans les autres UAFs. Toutefois, le taux de régénération global avec une prédominance de Detarium microcarpum est relativement plus élevé dans l'UAF 9 $(74,48 \%)$ suivi par celui des UAFs 1 et 8 (environ 65\%), et faible dans l'UAF 6 (14,02\%). Ainsi, Detarium microcarpum présente le meilleur taux de régénération dans les UAFs avec néanmoins un faible taux dans l'UAF 6 comme d'ailleurs pour les autres espèces étudiées qui ont des taux très faible (< 1\%). Crossopteryx febrifuga et Vitellaria paradoxa présentent des taux relativement faibles dans toutes les UAFs étudiées $(<4 \%)$ avec leurs meilleurs taux observés dans les UAFs 1 et 8 pour Vitellaria paradoxa, et dans les UAFs 9 et 8 pour Crossopteryx febrifuga. 
Les plus faibles taux sont observés chez Burkea africana dont la régénération est très faible dans l'UAF $9(0,48 \%)$ et nulle dans les autres UAFs.

\section{Distribution diamétrale des espèces}

Les répartitions en classes de diamètre des individus appartenant aux quatre espèces sont présentées dans les Figures 4 et 5. Les valeurs du paramètre de forme $\langle\mathrm{c} »$ de Crossopteryx febrifuga, Vitellaria paradoxa et de Detarium microcarpum varient très nettement suivant les UAFs étudiées contrairement à celles de Burkea african, qui sont presque similaires dans les UAFs. En effet, le coefficient de forme «c $»$ de Weibull est compris entre 1 et 3,6 pour Burkea africana dans toutes les UAFs, indiquant une distribution asymétrique positive caractérisée par une prédominance d'individus jeunes et de faible diamètre. Crossopteryx febrifuga présente également une distribution asymétrique positive dans les UAFs 1,6 et 8, alors que dans l'UAF 9, elle présente une distribution exponentielle décroissante $(\mathrm{c} \approx 1)$, caractéristique des populations à relativement fort potentiel de régénération mais présentant un problème de survie lors de la transition entre les stades de développement (Tableau 2). Dans les UAFs 1 et 6, Detarium microcarpum présente une distribution en $\mathrm{J}$ renversé $(c<1)$ tandis que dans les UAFs 8 et 9, sa distribution est exponentiellement décroissante $(\mathrm{c} \approx 1)$. Quant à Vitellaria paradoxa, sa distribution est asymétrique positive dans les UAFs 6 et 9 , et exponentiellement décroissante $(\mathrm{c} \approx 1)$ dans les UAFs 1 et 8.

L'examen de ces distributions diamétrales conformément à la loi de Weibull montre donc que Burkea africana et Crossopteryx febrifuga présentent chacune, une distribution asymétrique positive caractérisée par une prédominance d'individus de faible diamètre dans toutes les UAFs ainsi que Vitellaria paradoxa dans les UAFs 6 et 9 (Figures 4 et 5). Cependant, Detarium microcarpum présente des structures diamétrales en forme de $\mathrm{L}$ ou de $\mathrm{J}$ inversé sur l'ensemble des unités, surtout dans les UAFs 1 et 8 où les courbes de distribution de Vitellaria paradoxa présentent les mêmes allures; ce qui indique une forte présence de jeunes, mais également d'individus dont le développement pourrait être compromis par les strates arborescentes.

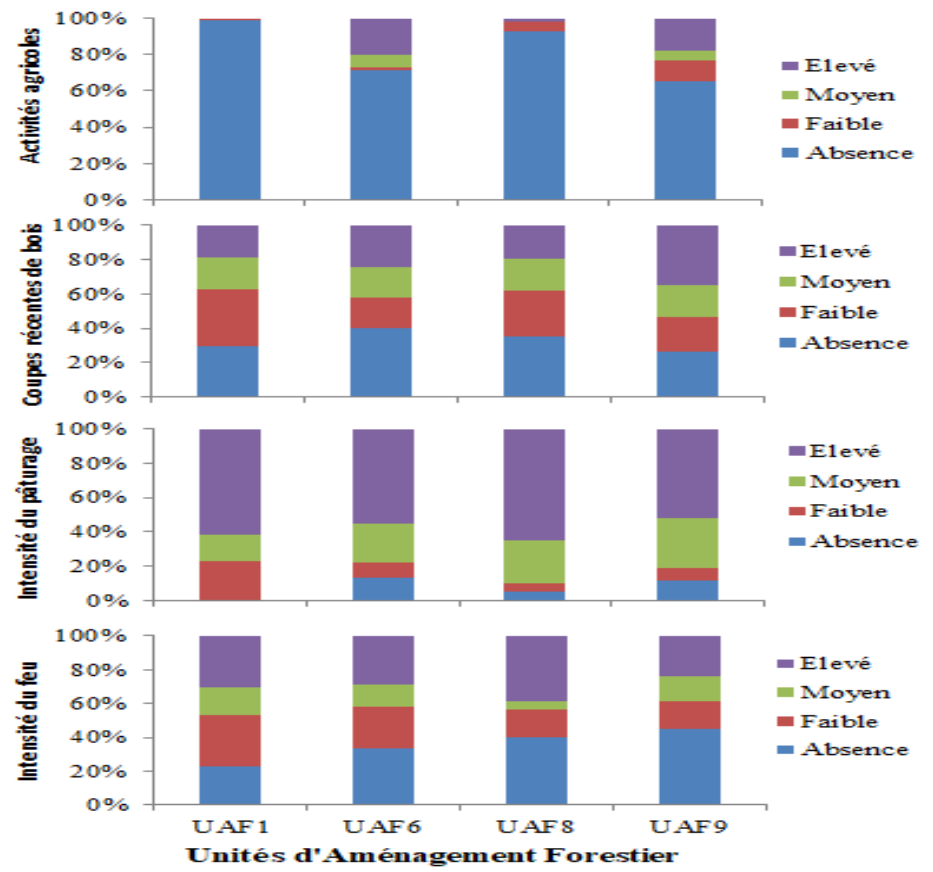

Figure 3: Intensités des différents facteurs anthropiques (activités agricoles, coupe de bois, pâturage et feu) en pourcentage (\%) de placettes affectées dans les UAFs étudiées. 
F. W. KAGAMBEGA et al. / Int. J. Biol. Chem. Sci. 13(6): 2666-2682, 2019

Tableau 3: Caractéristiques structurales des peuplements des espèces suivant les trois groupes de classes de diamètre dans les différentes UAFs.

\begin{tabular}{|c|c|c|c|c|c|c|c|c|c|c|c|c|c|}
\hline \multirow{2}{*}{$\begin{array}{l}\text { Classes de } \\
\text { diamètre }\end{array}$} & \multirow[b]{2}{*}{ Espèces recensées } & \multicolumn{3}{|c|}{ UAF 1} & \multicolumn{3}{|c|}{ UAF 6} & \multicolumn{3}{|c|}{ UAF 8} & \multicolumn{3}{|c|}{ UAF 9} \\
\hline & & $\begin{array}{l}\text { Densité } \\
\text { (ind/ha) }\end{array}$ & DBH (cm) & $\begin{array}{l}\text { Hauteur } \\
\text { (m) }\end{array}$ & $\begin{array}{l}\text { Densité } \\
\text { (ind/ha) }\end{array}$ & DBH (cm) & $\begin{array}{l}\text { Hauteur } \\
\text { (m) }\end{array}$ & $\begin{array}{l}\text { Densité } \\
\text { (ind/ha) }\end{array}$ & DBH (cm) & $\begin{array}{l}\text { Hauteur } \\
\text { (m) }\end{array}$ & $\begin{array}{l}\text { Densité } \\
\text { (ind/ha) }\end{array}$ & DBH (cm) & $\begin{array}{l}\text { Hauteur } \\
\text { (m) }\end{array}$ \\
\hline \multirow{5}{*}{$\begin{array}{l}\mathrm{DBH}<5 \\
\mathrm{~cm}\end{array}$} & Toutes les espèces & $1713,3 \mathrm{c}$ & $2,8 \pm 1,2 \mathrm{a}$ & $2,2 \pm 1,1 \mathbf{a}$ & $116,7 \mathrm{a}$ & $2,8 \pm 1,1$ a & $2,2 \pm 0,7 \mathrm{a}$ & $1308,3 \mathrm{~b}$ & $2,8 \pm 1,1$ a & $2,2 \pm 0,6 a$ & $1480,9 b$ & $2,5 \pm 1,1 \mathrm{a}$ & $2,1 \pm 0,7 \mathrm{a}$ \\
\hline & Buzbia quicasa & Néant & Néant & Néant & Néant & Néant & Néant & Néant & Néant & Néant & $9,5 \mathrm{~b}$ & $1,9 \pm 0,4 b$ & $1,7 \pm 0,1 b$ \\
\hline & $\begin{array}{l}\text { Crossaptenx } \\
\text { febrituga }\end{array}$ & $46,7 \mathrm{~b}$ & $3,7 \pm 1,1 \mathrm{a}$ & $2,7 \pm 1 \mathbf{a}$ & $6,7 \mathrm{a}$ & $3,7 \pm 0,6 \mathrm{a}$ & $2,6 \pm 0,2 \mathrm{a}$ & $50,0 \mathrm{~b}$ & $3,5 \pm 1,1 \mathrm{a}$ & $2,4 \pm 0,9 \mathrm{a}$ & $52,4 \mathrm{~b}$ & $2,7 \pm 1,4 \mathrm{a}$ & $2 \pm 0,9 \mathrm{a}$ \\
\hline & $\begin{array}{l}\text { Detarium } \\
\text { micrecarpum }\end{array}$ & $486,6 \mathrm{c}$ & $2,8 \pm 1,2 \mathrm{a}$ & $2,1 \pm 0,5 \mathrm{a}$ & 33,33 a & $2,6 \pm 1 \mathrm{a}$ & $1,9 \pm 0,4 \mathrm{a}$ & $308,3 \mathrm{~b}$ & $3,3 \pm 0,9 \mathrm{a}$ & $2,4 \pm 0,6 \mathrm{a}$ & $595,3 \mathrm{c}$ & $2,3 \pm 1,1 \mathbf{a}$ & $1,9 \pm 0,4 a$ \\
\hline & Kitallaria paxadoxa & $93,3 \mathrm{~d}$ & $2,8 \pm 1,2 \mathrm{a}$ & $1,9 \pm 0,5 \mathrm{a}$ & 2,2 a & $3,5 \pm 1,3 \mathrm{a}$ & $1,8 \pm 0,3 \mathrm{a}$ & $50,0 \mathrm{c}$ & $2,9 \pm 0,8 \mathrm{a}$ & $1,9 \pm 0,4 \mathrm{a}$ & $23,8 \mathrm{~b}$ & $2,7 \pm 1,3 \mathrm{a}$ & $1,9 \pm 0,5 \mathrm{a}$ \\
\hline \multirow{5}{*}{$\begin{array}{l}5 \mathrm{~cm} \leq \\
\mathrm{DBH}<10 \\
\mathrm{~cm}\end{array}$} & Toutes les espèces & $686,7 \mathrm{c}$ & $6,5 \pm 1,4 \mathrm{a}$ & $3,9 \pm 1,8$ a & $504,4 \mathrm{~b}$ & $6,3 \pm 1,6 \mathrm{a}$ & $4,1 \pm 1,7$ a & $523,3 \mathrm{~b}$ & $6,4 \pm 1,7 \mathrm{a}$ & $4,1 \pm 1,4 \mathrm{a}$ & $382,9 \mathrm{a}$ & $6,1 \pm 1,5$ a & $3,6 \pm 1,4 a$ \\
\hline & Burbsa quicoraa & $12 \mathrm{~b}$ & $\tau, 1 \pm 0,9 \mathbf{a}$ & $4,3 \pm 1,3 \mathrm{a}$ & $4,44 \mathrm{a}$ & $7,6 \pm 2,2 \mathrm{a}$ & $3,3 \pm 0,1 \mathrm{a}$ & $13,3 \mathrm{~b}$ & $7,6 \pm 1,6 \mathrm{a}$ & $4,8 \pm 1,9$ a & $3,8 \mathrm{a}$ & $6,3 \pm 0,9 \mathrm{a}$ & $3,9 \pm 1,1 \mathrm{a}$ \\
\hline & $\begin{array}{l}\text { Crossoptergx } \\
\text { febrifuga }\end{array}$ & $48 \mathrm{~b}$ & $6,5 \pm 1,3 \mathrm{a}$ & $3,7 \pm 0,9 \mathrm{a}$ & 6,67 a & $7,4 \pm 1,9 \mathrm{a}$ & $5,1 \pm 1,8$ a & $38,3 \mathrm{~b}$ & $6,4 \pm 1,8$ a & $4,8 \pm 1,3 \mathrm{a}$ & 13,3 a & $6,5 \pm 1,6 \mathrm{a}$ & $3,4 \pm 1,2 \mathrm{a}$ \\
\hline & $\begin{array}{l}\text { Detarium } \\
\text { microcarpum }\end{array}$ & $222,7 \mathrm{~b}$ & $6,5 \pm 1,4 \mathrm{a}$ & $3,8 \pm 1,1$ a & 95,56 a & $5,6 \pm 1,5 \mathrm{a}$ & $3,5 \pm 1,1 \mathrm{a}$ & $110,0 \mathrm{a}$ & $5,8 \pm 1,7 \mathrm{a}$ & $3,7 \pm 1,2 \mathrm{a}$ & 104,8 a & $6,0 \pm 1,3 \mathrm{a}$ & $3,7 \pm 1,1$ a \\
\hline & Vitellaria paradoxa & $74,7 \mathrm{c}$ & $6,7 \pm 1,2 \mathrm{a}$ & $3,6 \pm 1,1 \mathrm{a}$ & $8,89 \mathrm{a}$ & $7,4 \pm 0,8 \mathrm{a}$ & $3,3 \pm 0,9 \mathrm{a}$ & $68,3 \mathrm{c}$ & $6,9 \pm 1,5 \mathrm{a}$ & $3,9 \pm 1,1 \mathrm{a}$ & $24,7 \mathrm{~b}$ & $6,1 \pm 1,5 \mathrm{a}$ & $3,6 \pm 1,4 \mathrm{a}$ \\
\hline \multirow{5}{*}{$\begin{array}{l}\mathrm{DBH} \geq 10 \\
\mathrm{~cm}\end{array}$} & Toutes les espèces & $207,5 \mathrm{~b}$ & $16,5 \pm 7,1 \mathrm{a}$ & $7,1 \pm 2,8 \mathrm{a}$ & $195,11 \mathrm{~b}$ & $16,1 \pm 6,7 \mathbf{a}$ & $7,8 \pm 2,5 \mathrm{a}$ & $220,0 \mathrm{~b}$ & $17,5 \pm 7,2 \mathrm{a}$ & $8,1 \pm 3,7 \mathrm{a}$ & $124,5 \mathrm{a}$ & $18,8 \pm 8,7 \mathbf{a}$ & $7,5 \pm 2,6 \mathrm{a}$ \\
\hline & Burksa qficicana & $20,7 \mathrm{~b}$ & $19,6 \pm 6,2 \mathrm{a}$ & $9,1 \pm 3,1$ a & $12,67 \mathrm{a}$ & $19,4 \pm 6,7 \mathrm{a}$ & $9,2 \pm 2,6 \mathrm{a}$ & $12,3 \mathrm{a}$ & 22,2 a $\pm 7,5$ & $9,2 \pm 2,4 \mathrm{a}$ & $11,8 \mathrm{a}$ & $20,7 \pm 7,6 \mathrm{a}$ & $8,9 \pm 2,7$ a \\
\hline & $\begin{array}{l}\text { Crossopteryx } \\
\text { febrifuga } \\
\end{array}$ & $6,7 \mathrm{~b}$ & $11,5 \pm 1,6 \mathrm{a}$ & $4,8 \pm 1,1 \mathrm{a}$ & 3,33 a & $12,2 \pm 1,7 \mathrm{a}$ & $6,5 \pm 1,7$ a & $5,7 \mathbf{b}$ & $13,7 \pm 3,8 \mathrm{a}$ & $6,2 \pm 1,6 \mathrm{a}$ & $1,2 \mathrm{a}$ & $12,2=2,8 \mathrm{a}$ & $6,5 \pm 1,3 \mathrm{a}$ \\
\hline & $\begin{array}{l}\text { Detarium } \\
\text { microcarpum } \\
\end{array}$ & $22 \mathrm{c}$ & $11,8 \pm 2,2 \mathrm{a}$ & $5,3 \pm 1,3 \mathbf{a}$ & $14,00 \mathrm{~b}$ & $12,7 \pm 3,4 \mathrm{a}$ & $6,7 \pm 1,8$ a & $10,2 \mathrm{~b}$ & $12,3 \pm 2,5 \mathrm{a}$ & $6,6 \pm 2,2 \mathrm{a}$ & $4,3 \mathrm{a}$ & $11,9 \pm 2,4 \mathrm{a}$ & $4,9 \pm 0,9 \mathrm{a}$ \\
\hline & Vitsllapia paradioxa & $37,9 \mathrm{~b}$ & $17,2 \pm 7,1 \mathbf{a}$ & $6,8 \pm 2,4 \mathrm{a}$ & $25,33 \mathrm{a}$ & $19,1 \pm 7,7 \mathbf{a}$ & $7,1 \pm 2,1 \mathrm{a}$ & $54,2 \mathrm{c}$ & $17,5 \pm 6,3 \mathrm{a}$ & $7,1 \pm 3,9 \mathrm{a}$ & $31,4 \mathrm{a}$ & $20,8 \pm 9,1 \mathrm{a}$ & $7,1 \pm 2,2 \mathrm{a}$ \\
\hline
\end{tabular}

DBH = Diamètre à hauteur de poitrine $(1,30 \mathrm{~m})$.

Pour chaque espèce, les valeurs suivies des lettres différentes dans la même ligne sont significativement différentes suivant les UAFs. 
Tableau 4: Variation du potentiel de régénération naturelle des espèces dans les différentes UAFs étudiées.

\begin{tabular}{ccccc}
\hline Espèces & UAF 1 & UAF 6 & UAF 8 & UAF 9 \\
\hline Burkea africana & 0,00 & 0,00 & 0,00 & 0,48 \\
Crossopteryx febrifuga & 1,79 & 0,82 & 2,44 & 2,64 \\
Detarium microcarpum & 18,66 & 4,08 & 15,03 & 29,94 \\
Vitellaria paradoxa & 3,58 & 0,27 & 2,44 & 1,20 \\
Toutes les espèces recensées & 65,71 & 14,02 & 63,77 & 74,48 \\
\hline
\end{tabular}

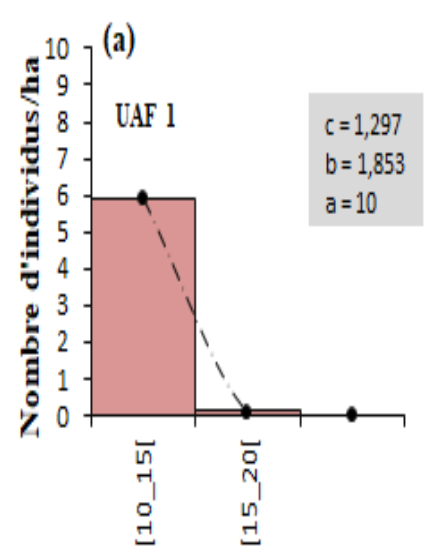

Classe de diamètre $(\mathrm{cm})$

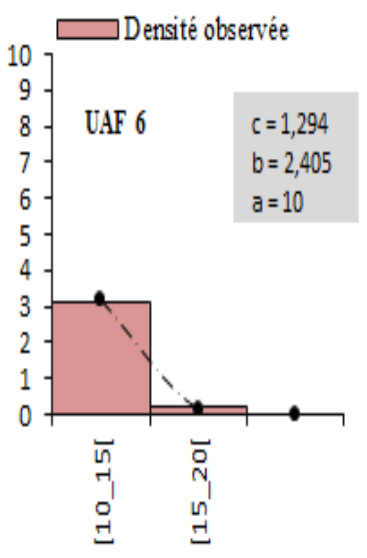

Classe de diamètre $(\mathrm{cm})$

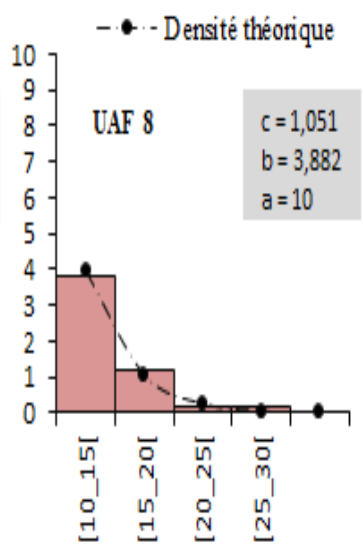

Classe de diamètre $(\mathrm{cm})$

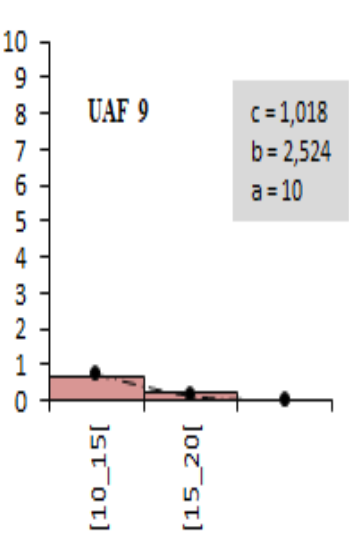

Classe de diamètre $(\mathrm{cm})$

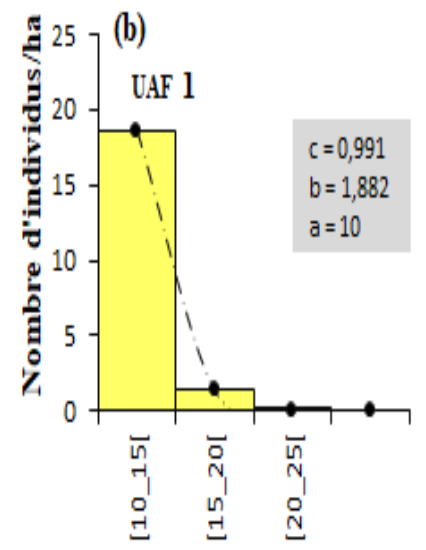

Classe de diamètre $(\mathrm{cm})$

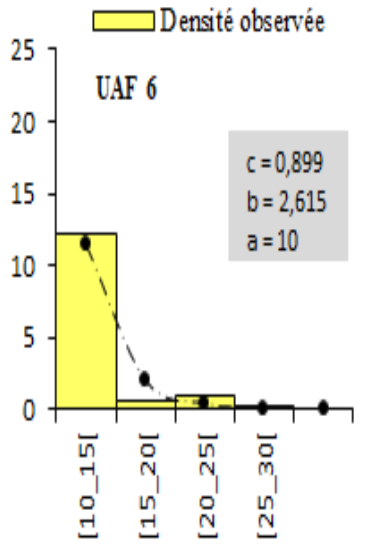

Classe de diamètre $(\mathrm{cm})$

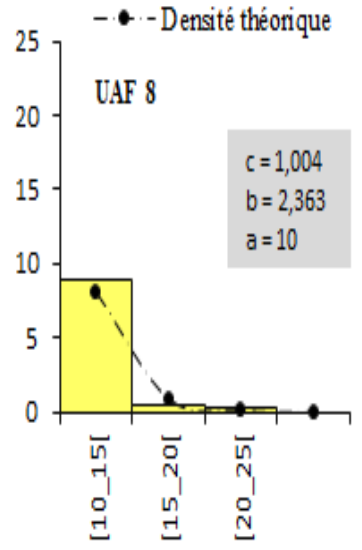

Classe de diamètre $(\mathrm{cm})$

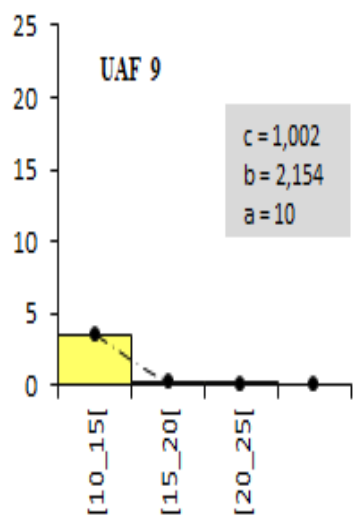

Classe de diamètre $(\mathrm{cm})$

Figure 4 : Distribution des classes de diamètre pour les individus de $\mathrm{DBH} \geq 10 \mathrm{~cm}$ de Crossopteryx febrifuga (a) et de Detarium microcarpum (b) dans les unités d'aménagement forestiers (UAF). 


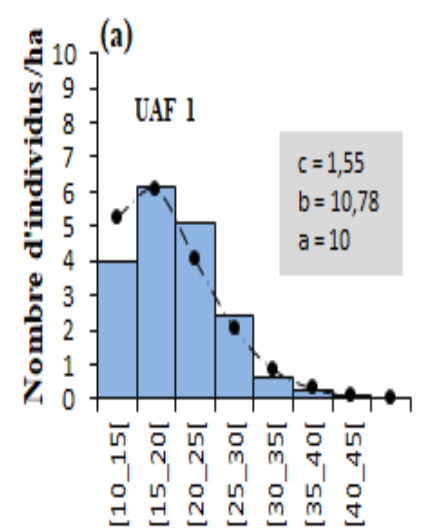

Classe de diamètre $(\mathrm{cm})$

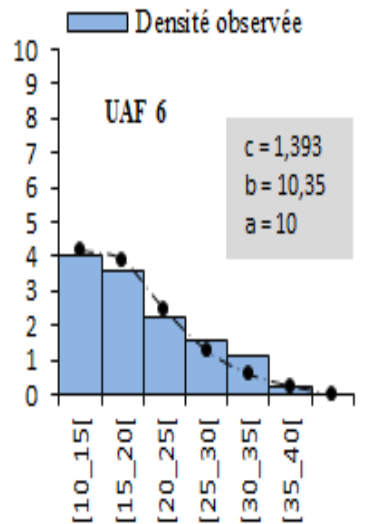

Classe de diamètre $(\mathrm{cm})$

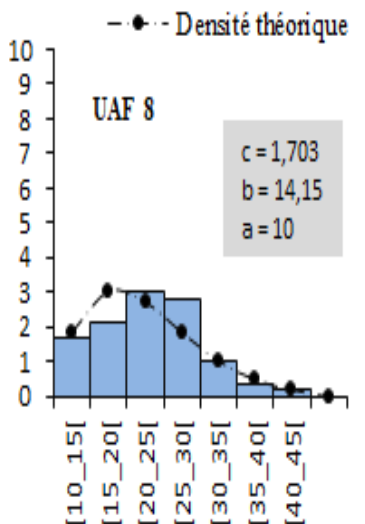

Classe de diamètre $(\mathrm{cm})$

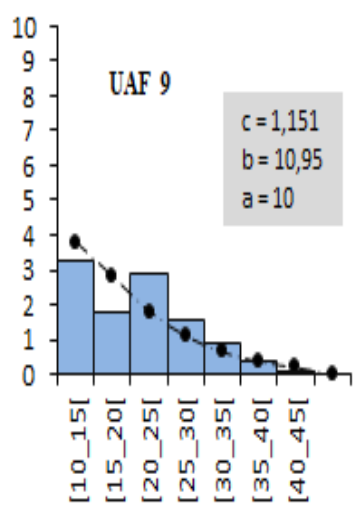

Classe de diamètre $(\mathrm{cm})$
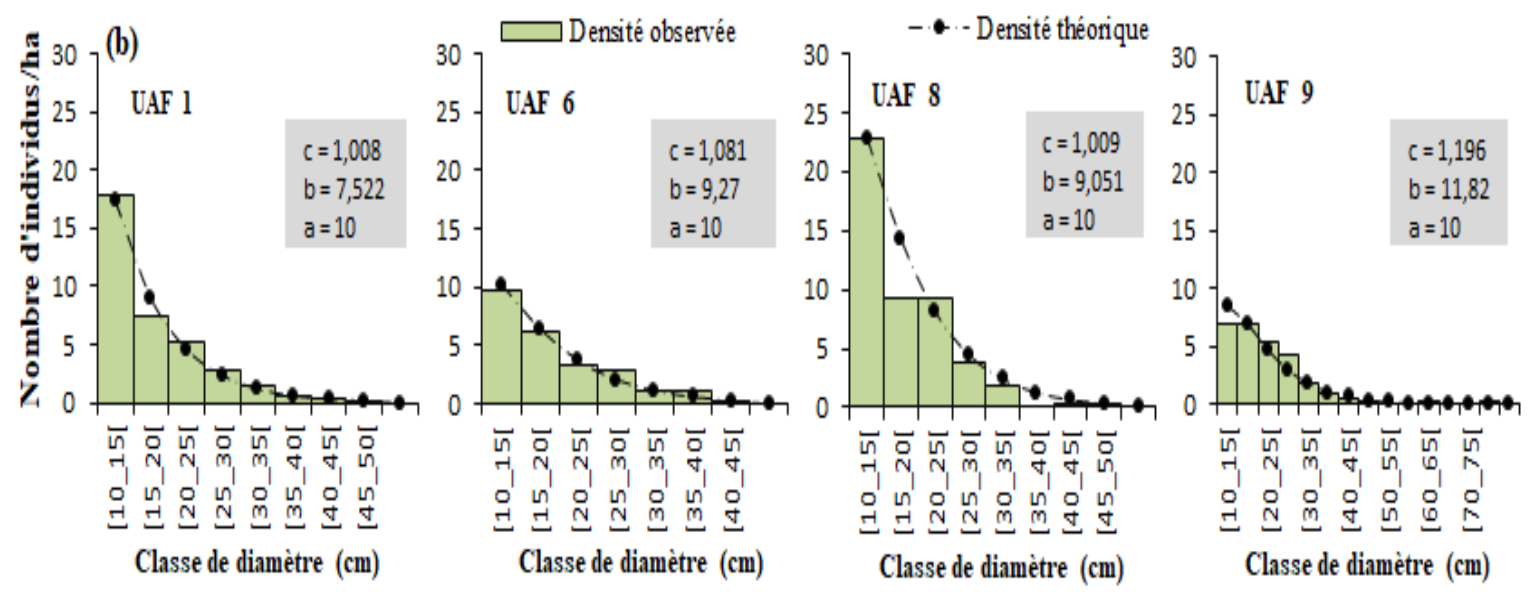

Figure 5: Distribution des classes de diamètre pour les individus de $\mathrm{DBH} \geq 10 \mathrm{~cm}$ de Burkea Africana (a) et de Vitellaria paradoxa (b) dans les unités d'aménagement forestiers (UAF).

\section{DISCUSSION}

\section{Ampleur des facteurs de perturbation anthropiques dans les UAFs étudiées}

Les résultats sur la perturbation anthropique montrent qu'en dehors des activités agricoles qui sont relativement moins fréquentes, les autres facteurs anthropiques (coupes frauduleuses de bois, feux de végétation et pâturage) ont été très conséquents dans les UAFs étudiées (Figure 3). L'UAF 1 qui ne présente aucune trace d'activité agricole, a été le plus perturbé par le feu et le pâturage suivi par l'UAF 8 , tandis que l'impact de l'agriculture a été plus important dans les UAFs 6 et 9 plus éloignés de l'organe de gestion. L'inexistence de champs dans l'UAF 1 peut s'expliquer par sa situation dans la localité de Cassou qui abrite l'unité chargée de la surveillance du CAF; cette proximité $(3 \mathrm{~km})$, fait qu'elle bénéficie d'une forte surveillance administrative l'épargnant des activités agricoles. Toutefois, des investigations conduites par Yoni et Sedogo (2015), indiquent que le contrôle du service technique et forestier est insuffisant. Les incidences élevées du feu et du pâturage dans cette même UAF 1 malgré sa proximité à l'unité gestion, révèlent donc des failles dans le dispositif de surveillance mis en place. En effet, les prescriptions prévues dans le cahier de charge, en matière d'administration des feux précoces et d'interdiction du pâturage, sont rarement respectées du fait du manque de suivi et de surveillance des parcelles 
(Ouédraogo, 2004). De plus, la proximité de l'UAF 1 à Cassou et ces villages riverains, constitue également une opportunité de ravitaillement en ressource fourragère par le bétail airant ou les pasteurs transhumants. Il ressort également que toutes les UAFs étudiées sont affectées par la coupe récente de bois et que les UAFs 9 et 1 sont les plus touchées avec une incidence plus élevée dans l'UAF 9. Ces résultats corroborent ceux obtenus par Yoni et Sedogo (2015) et Sawadogo (2006) qui expliquent ces faits par le non-respect des prescriptions de l'aménagement et l'insuffisance de la surveillance du service technique et forestier dans les chantiers du Centre- Ouest.

\section{Impact des pressions anthropiques sur la densité et la structure démographique des espèces}

La structure démographique des quatre espèces (Burkea africana, Detarium microcarpum, Crossopteryx febrifuga et Vitellaria paradoxa) montre que la densité diminue avec l'augmentation du diamètre (DBH $<5 \mathrm{~cm}$ vers $\mathrm{DBH} \geq 10 \mathrm{~cm}$ ) dans les UAFs 1 et 8 . Ces résultats montrent une tendance de dynamique progressive (Thiombiano, 2005) et indiquent que l'ensemble des peuplements de ces espèces présente un bon état. Dans ce sens, le système d'exploitation et les facteurs de perturbation n'ont pas eu d'effet négatif sur la structure de l'ensemble des espèces étudiées dans les UAFs 1 et 8 . Par contre dans les UAFs 6 et 9 , les espèces présentent des structures avec une prédominance des individus à gros diamètre $(\mathrm{DBH} \geq 10 \mathrm{~cm})$ de Vitellaria paradoxa et de Burkea africana dans l'UAF 6 et une prédominance des individus à diamètres moyens $(5 \mathrm{~cm} \leq \mathrm{DBH}<10 \mathrm{~cm})$ pour Detarium microcarpum et Vitellaria paradoxa respectivement dans l'UAF 6 et l'UAF 9. La situation des espèces dans les UAFs 6 et 9 qui sont les plus éloignées de l'unité de gestion, peut s'expliquer par l'existence et l'abondance des facteurs de perturbations que sont le feu, le pâturage et la coupe frauduleuse qui les affectent (Boussim et al., 2009). Toutefois, Detarium microcarpum présente un histogramme de distribution en allure de « $\mathbf{J}$ renversé » globalement dans les quatre UAFs; ce qui indique que cette espèce a un potentiel pour reconstituer des populations stables dans ces UAFs. Par contre, les allures en « cloche » des histogrammes de distribution de Burkea africana dans toutes les UAFs, traduisent des peuplements perturbés. L'explication réside évidement dans la recherche de bois de chauffe dans les environnements immédiats des villages (Maazou et al., 2017) et surtout par le fait que ces UAFs font l'objet d'une exploitation commerciale de bois en direction des centres urbains. La perturbation des peuplements illustrée par la faible proportion des individus de petites classes de diamètre est plus accentuée dans les UAFs 6 et 9 ; cela s'explique par le non-respect des prescriptions d'aménagement et l'insuffisance de la surveillance dans ces UAFs (Yoni et Sedogo, 2015) du fait de leur situation éloignée par rapport à l'unité de gestion.

L'une des principales causes de régression des formations ligneuses est la raréfaction des jeunes individus susceptibles de remplacer les sujets vieillissants (Ouédraogo et al., 2006). Les résultats de la présente étude montre qu'à l'exception de l'UAF 6 où la régénération est très faible, les individus jeunes de l'ensemble des espèces (diamètre inférieur à $5 \mathrm{~cm}$ ) sont mieux représentés dans les autres UAFs avec les meilleurs taux pour Detarium microcarpum et des faibles taux de régénération $(<5 \%)$ pour Crossopteryx febrifuga et Vitellaria paradoxa. Les plus faibles taux ont été observés chez Burkea africana avec une régénération très faible dans l'UAF $9(0,48 \%)$ et nulle dans les autres UAFs. Selon Nacoulma (2005), les faibles taux de régénération des espèces sont la conséquence des coupes frauduleuses de bois ainsi que des feux de brousses répétés et incontrôlés qui ne laissent pas suffisamment de temps aux espèces pour se reconstituer, justifiant ainsi nos résultats. Des observations analogues ont été faites par Boussim et al. (2009) dans le CAF de Cassou et par Sawadogo (2007) dans le CAF du Nazinon, qui ont constaté également que Crossopteryx febrifuga et Burkea africana étaient menacées 
de disparition à cause des problèmes de régénération (quasi nulle). Une faible représentation d'individus juvéniles a été observée chez Vitellaria paradoxa surtout dans les UAFs 6 et 9 ; cette situation peut s'expliquer par les pressions intenses de pâturage et de feu, exercées sur les formations végétales de ces UAFs du fait de leur situation éloignée de l'unité de gestion du CAF. Cette remarque rejoint les observations de Bokary et al. (2004) et de Dan Guimbo et al. (2010) qui expliquent ces difficultés d'affranchissement des individus juvéniles de Vitellaria paradoxa par les effets néfastes des feux de brousse qui ne favorisent pas l'évolution des plantules qui apparaissent. Par ailleurs, les feux précoces ou d'aménagement peuvent aussi entraîner une mortalité très élevée des jeunes individus de l'ensemble des espèces. La bonne régénération de Detarium microcarpum et sa structure stable dans les différentes UAFs malgré les facteurs de dégradation, corroborent les observations de Kaboré (2005) et de Sawadogo (2007) dans d'autres CAF du Burkina Faso, et s'expliquent par la grande capacité de drageonnage de l'espèce qui constitue un atout de bonne régénération car les drageons ont une croissance plus rapide que les semis (Bellefontaine et al., 2000). Ces résultats s'expliquent également par le fait que Detarium microcarpum présente au stade juvénile une bonne capacité d'adaptation aux principaux facteurs traumatisants du milieu, tels que la sécheresse, les feux et la coupe répétée (Sawadogo, 2006).

\section{Conclusion}

Cette étude a permis de caractériser l'ampleur des facteurs anthropiques et de décrire la structure du peuplement des quatre espèces ciblées, dans les unités d'aménagements forestiers. En dehors des activités agricoles qui ont été relativement peu fréquentes dans les UAFs étudiées, les autres facteurs de perturbation anthropiques ont été fréquents avec des ampleurs variables d'une UAF à l'autre. Les peuplements de Detarium microcarpum et dans une moindre mesure de Vitellaria paradoxa présentent une bonne dynamique structurale dans toutes les UAFs malgré les perturbations anthropiques. Ces deux espèces pourraient assurer une exploitation durable sauf que pour Vitellaria paradoxa, qui est une espèce protégée, il serait mieux indiqué que l'exploitation de son bois soit uniquement dirigée vers les sujets malades ou morts. La dynamique des peuplements de Crossopteryx febrifuga apparaît satisfaisante seulement dans l'UAF 8 et son exploitation pourrait compromettre sa présence dans les autres UAFs si des mesures spécifiques de conservation ne sont pas prises. La structure de Burkea africana révèle un faible potentiel de régénération et une faible abondance d'individus adultes dans toutes les UAFs ; il serait donc judicieux de limiter son exploitation afin d'éviter la disparition de l'espèce dans le CAF. Partant des hypothèses de départ, les résultats obtenus montrent qu'à l'exception du pâturage, les UAFs proches de l'unité de gestion ont été moins perturbées par les autres facteurs; ce qui confirme partiellement la première hypothèse. Cependant, la deuxième hypothèse est rejetée en partie car en dehors de Detarium microcarpum les autres espèces ont présenté des structures instables mêmes dans toutes les UAFs proches de l'unité de gestion.

\section{CONFLIT D'INTERETS}

Les auteurs de ce manuscrit déclarent qu'il n'y a aucun conflit d'intérêts.

\section{CONTRIBUTIONS DES AUTEURS}

Dans la réalisation de la présente étude, FWK et BZN ont avec l'appui de LS, élaboré le protocole de recherche et collecté les données de terrain. AK et $\mathrm{NZ}$ ont appuyé FWK dans le traitement des données et ont participé activement à la rédaction du manuscrit. LS et JIB ont participé à la relecture et à la finalisation du manuscrit.

\section{REMERCIEMENTS}

Les auteurs remercient $\mathrm{Mr}$ Michael Philippe Bessike BALINGA, pour sa collaboration et son assistance logistique ainsi que $\mathrm{Mr}$ Abdoulaye RABDO pour son appui dans la réalisation des cartes. 


\section{REFERENCES}

Aboubacar K, Douma S, Moussa Mamoudou B, Djermakoye Seyni RS. 2018. Structure des populations naturelles de Neocarya macrophylla (Sabine) Prance, ligneux d'intérêt alimentaire, dans le Dallol Bosso (Niger). Bois et Forêts des Tropiques, 337: 67-78. DOI: https://doi.org/10.19182/bft2018.337.a31 630

Agbani PO, Amagnide A, Cédric Goussanou C, Azihou F, Sinsin B. 2018. Structure des peuplements ligneux des formations végétales de la forêt sacrée de Nassou en zone soudanienne du Bénin. Int. J. Biol. Chem. Sci., 12 (6): 2519-2534. DOI: https://dx.doi.org/10.4314/ijbcs.v12i6.5

Angiosperm Phylogeny Group (APG) III. 2009. An update of the Angiosperm Phylogeny Group classification for the orders and families of flowering plants. Botanical Journal of the Linnean Society, 161 (2): 105-121. DOI: https://doi.org/10.1111/j.10958339.2009.00996.x

Arevalo J. 2016. Improving wood fuel governance in Burkina Faso: The experts' assessment. Renewable and Sustainable Energy Reviews, 57: 1398 1408.

DOI: https://doi.org/10.1016/j.rser.2015.12.17 8

Bellefontaine R, Edelin C, Ichaou A, du Laurens D, Monsarrat A, Loquai C. 2000. Le drageonnage, alternative aux semis et aux plantations de ligneux dans les zones semi-arides: protocole de recherches. Sécheresse, 11 (4): 221-226.

Bokary AK, Bouvet JM, Picard N. 2004. Size class distribution and spatial pattern of Vitellaria paradoxa in relation to farmer practices in Mali. Agroforestry Systems, 60: 3-11.

DOI: 10.1023/B:AGFO.0000009400.24606.e3

Bouda H, Savadogo P, Tiveau D, Ouedraogo B. 2011. State, forest and community: Challenges of democratically decentralizing forest management in the
Centre-West Region of Burkina Faso. Sustainable Development, 19 (4): 275288. DOI: https://doi.org/10.1002/sd.444 Boussim IJ, Ouedraogogo A, Lankoandé B. 2009. Etude des impacts écologiques dans les unités d'aménagements forestiers des régions du Centre-Nord et du centre-Ouest. In Bois-énergie au Burkina Faso : Consolidation Des Moyens d'Existence Durable (2006 2009), Kabré AM, Somda J, Savadogo M, Nianogo AJ (eds).Ouagadougou, Burkina Faso ; 115-139.

Coulibaly-Lingani P, Savadogo P, Tigabu M, Odén PC. 2011. Factors influencing people's participation in the forest management program in Burkina Faso, West Africa. Forest Policy and Economics, 13 (4): 292-302. DOI: 10.1016/j.forpol.2011.02.005.

Coulibaly-Lingani P, Tigabu M, Savadogo P, Odén PC. 2014. Participatory forest management in Burkina Faso: Members' perception of performance. Journal of Forestry Research, 25 (3): 637-646. DOI: 10.1007/s11676-014-0502-x.

Dan Guimbo I, Mahamane A, Ambouta KJM. 2010. Peuplement des parcs à Neocarya macrophylla (Sabine) Prance et à Vitellaria paradoxa (Gaertn. C.F.) dans le sud-ouest nigérien : diversité, structure et régénération. Int. J. Biol. Chem. Sci., 5 (4): 1706 - 1720. DOI: 10.4314/ijbcs.v4i5.65568.

Driessen P, Deckers J, Spaargaren O. 2001. Lectures Notes on the Major Soils of the World. In: FAO World Soil Resources, Report-94. Rome, Italy, Food and Agriculture Organization of the United Nations.

Dro B, Soro D, Kone MW, Bakayoko A, Kamanzi K. 2013. Evaluation de l'abondance de plantes médicinales utilisées en médecine traditionnelle dans le Nord de la Côte d'Ivoire. Journal of Animal \& Plant Sciences, 17 (3): 2631 2646.

DOI: 
http://www.m.elewa.org/JAPS; ISSN 2071-7024.

Etongo D, Kanninen M, Epule Epule T, Fobissie K. 2018. Assessing the effectiveness of joint forest management in Southern Burkina Faso: A SWOTAHP analysis. Forest Policy and Economics, 90: 31-38. DOI: 10.1016/j.forpol.2018.01.008.

FAO, 2015. Global forest resources assessment. Food and Agriculture Organization of the United Nations, Rome, 253p.

Gbedahi OLC, Biaou SSH, Mama A, Gouwakinnou GN, Yorou NS. 2019. Dynamique du couvert végétal à Bassila au nord Bénin pendant et après la mise en œuvre d'un projet d'aménagement forestier. Int. J. Biol. Chem. Sci., 13 (1): 311-324.

DOI: https://dx.doi.org/10.4314/ijbcs.v13i1.25

Glèlè Kakaï R, Bonou W, Lykke AM. 2016. Approche méthodologique de construction et d'interprétation des structures en diamètre des arbres. Annales des Sciences Agronomiques (spécial Projet Undesert-UE), 20: 99112. ISSN 1659-5009.

Kaboré C. 2005. Aménagement des forêts au Sahel: point sur vingt années de pratiques au Burkina Faso, 125p.

Kouyate AM, Lamien N. 2011. Conservation et utilisation durable des ressources génétiques des espèces ligneuses alimentaires prioritaires de l'Afrique subsaharienne. INERA, Centre Régional de Recherches Environnementales et Agricoles, Burkina Faso, 8p.

Maazou R, Rabiou H, Issiaka Y, Abdou L, Idi Saidou S, Mahamane A. 2017. Influence de l'occupation des terres sur la dynamique des communautés végétales en zone Sahélienne : cas de la commune rurale de Dantchandou (Niger). Int. J. Biol. Chem. Sci., 11 (1): 79-92. DOI : http://dx.doi.org/10.4314/ijbcs.v11i1.7

MEDD (Ministère de l'Environnement et du Développement Durable), 2011.
Programme d'Investissement Forestier Burkina Faso, volume 2, 130p.

Nacoulma BMI. 2005. Etude des espèces ligneuses utilisées en médecine traditionnelle vétérinaire dans la zone de Nobéré (Burkina Faso). Mémoire de DEA. Université de Ouagadougou, Burkina Faso, 74p.

Ouédraogo K. 2004. Suivi des impacts socioéconomiques et écologiques du programme RPTES composante écologique. Atelier de capitalisation du projet RPTES Phase initiale, 11 et 12 Novembre 2004, Koudougou, Burkina Faso, $17 \mathrm{p}$.

Ouédroago A, Thiombiano A, Hahn-Hadjali K, Guinko S. 2006. Diagnostic de l'état de dégradation des peuplements de quatre espèces ligneuses en zone soudanienne du Burkina Faso. Sécheresse, 17 (4): 485-491. DOI: $10.1684 / \mathrm{sec} .2006 .0058$

R Development Core Team, 2008. A language and environment for statistical computing. R Foundation for Statistical Computing, Vienna, Austria.

Sapkota IP, Odén PC. 2009. Gap characteristics and their effects on regeneration, dominance and early growth of woody species. Journal of plant ecology, 2 (1): 21-29. DOI: https://doi.org/10.1093/jpe/rtp004.

Sawadogo L. 2006. Adapter les approches de l'aménagement durable des forêts sèches aux aptitudes sociales, économiques et technologiques en Afrique. Le cas du Burkina Faso. Center for International Forestry Research, 59p.

Sawadogo L. 2007. Etat de la biodiversité et la de production des ligneux du Chantier d'Aménagement Forestier du NAZINON après une vingtaine d'années de pratiques d'aménagement. Center for International Forestry Research, Bogor, Indonesia, 42p.

Sawadogo L. 2009. Influence des facteurs anthropiques sur la dynamique de la végétation des forêts classées de Laba et 
de Tiogo en zone soudanéenne au Burkina Faso. Thèse doctorat d'Etat, Université de Ouagadougou, Burkina Faso, 142p.

Sereme A, Millogo-Rasolodimby J, Guinko S; Nacro M. 2008. Propriétés thérapeutiques des plantes à tanins du Burkina Faso. Pharmacopée et Médecine traditionnelle Africaines, 15: 41-49.

Sorg J-P. 2000. La durabilité en foresterie: pour une transformation durable de l'action forestière. Schweiz. Z. Forstwes, 151 (12): 522-525. DOI: 10.3188/szf.2000.0522.

Stohlgren TJ, Falkner MB, Schell LD. 1995. A modified-Whittaker nested vegetation sampling method. Vegetatio, 4: 1-8. DOI:

http://dx.doi.org/10.1007/BF00045503

Tanyi TF, Etongo D, Rabdo A. 2018. Assessing the sustainability of fuelwood production and its potential impact on
REDD+ in Burkina Faso. Int. Journal of Environmental Studies, 75 (1): 186-200. DOI :

https://doi.org/10.1080/00207233.2017.1 386435.

Thiombiano A, 2005. Les Combretaceae du Burkina Faso. Taxonomie, écologie, dynamique et régénération. Thèse d'Etat, Université de Ouagadougou, Burkina Faso. 295p.

Yoni G, Sedego S. 2015. Rapport final mission d'évaluation technique, socioéconomique, écologique et de gouvernance des Chantiers d'Aménagement Forestier (CAF) de Bougnounou -Nebielianayou, de Cassou, du Nazinon et de SapouyBieha dans la région du Centre Ouest au Burkina Faso. Ministère de l'Environnement et des Ressources Halieutiques. Initiatives Conseils International. 101. 